


\section{Deploying Expert Systems in Ada}





\section{Preface}

This research was conducted under auspices of the Research Institute for Computing and Information Systems by Inference Corporation. Dr. Charles McKay served as RICIS research coordinator.

Funding has been provided by the Information Systems Directorate, NASA/JSC through Cooperative Agreement NCC 9-16 between the NASA Johnson Space Center and the University of Houston-Clear Lake. The NASA technical monitor for this activity was Robert T. Savely, of the Software Technology Branch, Information Technology Division, Information Systems Directorate, NASA/JSC.

The views and conclusions contained in this report are those of the authors and should not be interpreted as representative of the official policies, either express or implied, of NASA or the United States Government. 



\title{
Deploying Expert Systems in Ada
}

\author{
S. Daniel Lee and Bradley P. Allen' \\ Inference Corporation \\ $5300 \mathrm{~W}$. Century Blyd \\ I.os Angeles, C.A 90045

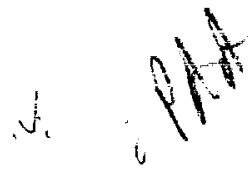

\section{Abstract}

As the Department of Defense Ada mandate begins to be enforced actively, interest in deploying expert systems in Ada has increased. This paper introduces a prototype Adabased expert system tool called ART/Ada. This protolype was built to support research into the language and operational issues of expert systems in Acla. ART Ada allows applications of a conventional expert system tool called ART-IM (Automated Reasoning Tool for Information Management) to be deployed in various Ada environments with efficient use of time and space. ART-M, a C-based expert system tool, is used to generate Ada source cole which is compiled and linked with an Ada-based inference engine to produce an Ada execurable image. The future research directions call for improved support for real-time embedded and distributed expert systems. ART/Ada will be used to implement several prototype expert systems for the Space Station Freedom Program testbeds.

\section{Introduction}

\subsection{Motivation}

As the Department of Defense mandate to standardize on Ada as the language for embedded software systems development begins to be actively enforced, interest from developers of large-scale Ada systems in making expert systens technology readily available in Ada environments has increased.

Two examples of Ada applications that can benefit from the use of expert systems are monitoring and control systems and decision support systems Monitoring and control syitems demand real-time performance, small execution images, tight integration with other applications, and predictable demands on processor resources; decision support systems have somewhat less stringent requirements. An example project that exhibits the need for both of these types of systems is NASA's space station Freedom. Monitoring and control systems that will perform fault detection, isolation and reconfiguration for various on-board systems are expected to be developed and deployed on the station either in its initial operating configuration or as the station evolves: decision support systems that will provide assistance in activities such as crew-time scheduling and failure mode analysis are also under consideration. These systems will be expected to run reliably on a standard data processor, currently efvisioned to be a $1-16$ megabyte 80386-based workstation. The Station is typical of the large Ada soltware development projects that will require expert systems in the 1990 's.

Another large-scale application that can be benefited from the Ada-based expert system lool technology is the Pilot's Associate (PA) expert system project for military combat aircraft [9]. Funcled by the Defense Advanced Research Projects Agency (D.ARPA) as part of its Strategic Computing Program, the PA project attempts to automate the cockpit of military combat aircraft using Artificial Intelligence (AI) technicues. A Lisp-based expert system rool, ART (Aulomated Reasoning Tool), was used to implement the Phase I

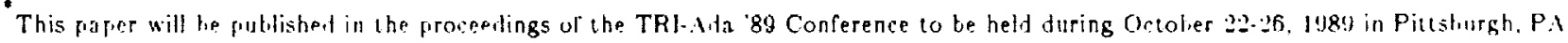


protolype An tiabased expert stivem tool can provide a migration path to deploy the protogye on an on-board computer berause da cross-compilers are readily arailable to run tha programs on most embedeled processors used for avionics.

\subsection{Approach}

Inference Corporation dereloped an expert system tool called ART (Automated Reasoning Tool) that has been commercially arailable for several years 11 !. ART is writen in Common Lisp and it supports various reasoning facilities such as rules, objects, truth maintenance. hypothetical reasoning and objectoriented programming. Last year, Inference introduced another expert system tool called ART-IM Automated Rensoning 'Tool for Information Management), which is also commercially available $[12] . \quad A R T-I M$ is written in $\mathrm{C}$ and it supports a major subset of AR'T's leasoning facilities including rules, objects, truth maintenance and object-oriented programming Both ART and ARTMI have been successfully used to develop many applications which are in daily use today $\{5],\{16] .[17\}$.

Our approach in designing a prototype Ada-based expert system tool was to use the architecture of proven expert system lools: ART and ART-IM. ART$M$ was selected as a baseline system because $C$ is much closer to Ada. While ART-MM's inference engine was reimplemented in Ada, ART-IM's frontend (its parserianalyzer and user interface) was not. Instead. ART-M was enhanced to generate Ada source code that would be used to initialize Ada data structures equivalent to ART-MI's internal C data structures. This approach allows the user to take full advantage of . ART-M's interactive development environment while developing an application; once the development is complete. the application is converted 10. Ada source code that is compiled and linked with the Ada runtime kernel.

\subsection{The ART-IM Expert System Tool}

ART-M is a general purpose expert system tool written in $C$. It consists of

- a runtime kernel.
- a C deployment compiler. and

- an interactive development unvironment

ART-RM's kernel supports the following reames:

- a forward-chaining rule srielem hased on the Rete algorithm 6 !.

- an object system.

- object-oriented programming.

- a justilication-based truth maintenance system(JTMS), and

- explanation generation utilities.

ART-M supports deployment of applications in C using a $\mathrm{C}$ deployment compiler that converts an application into $\mathrm{C}$ data structure definitions in the form of either $\mathrm{C}$ source code or object code

ART-M's interactive development environment includes a highly functional user interface that allows browsing and debugging of the knowtedge base and an integrated editor that allows incremental compilation

ART-M is available for VMS. MNS and MS-DOS environments.

\section{ART/Ada: An Ada-based Expert System Tool}

\subsection{Ada Runtime Kernel}

The ART/Ada runtime kernel is composed of the following components:

- an inference engine.

- a procedural interface pactiage,

- a memory managenent package. and

- Ada deployment compiler utilities.

ART/Ada's inference engine is an Ada implementation of $A R^{\prime} \Gamma-\Gamma I^{\circ}$ s inference engine and is 
lunctionally identical to ART-Mls.

ART Ada's procedural interface includes all public functions in ART-IM except for lhose that are nsed only during the development phase and those that are part of ART-CI's user interface toolkit. ART Ada: procedural interlace can be used rither in the righthand side of a rule. or dirersly in user's tda plograms. The procedural inlerface includes data type conversions between the Ada data lypes and the ART-IM data types, predicates, operations on ARTM objects, ART-MT commands, I O functions and math functions.

ART Ada's memory management package uses the Ada features new and unchecked deallocation to allocate and deallocate memory.

The ART/Ada runtime kernel contains utilities called by the Ada code generated by the Ada deployment compiler.

\subsection{Ada Deployment Compiler}

ART-IM was augmented with an Ada deployment. compiler to support ART/Ada. As shown in ligure $2-1$, its input is an ART-M source file, and its output is Ada source liles. At any point alter an ART-IM source file is loaded into ART-M, and the knowledge base is initialized for execution, the Ada deployment compiler may be invoked to generate the Ada source code that would initialize the internal data structures of ART/Ada. An Ada package specification generated by ART-IM for an example application called MY_EXPERT_SYSTEM is as follows:

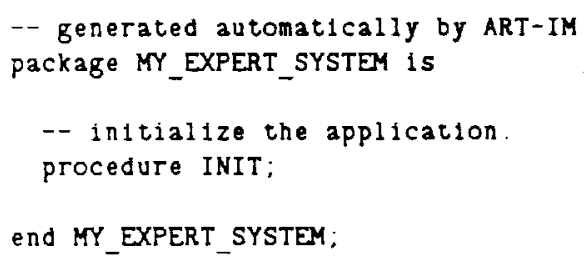

An Ada main program that the user would write to initialize and run the application would look like this:

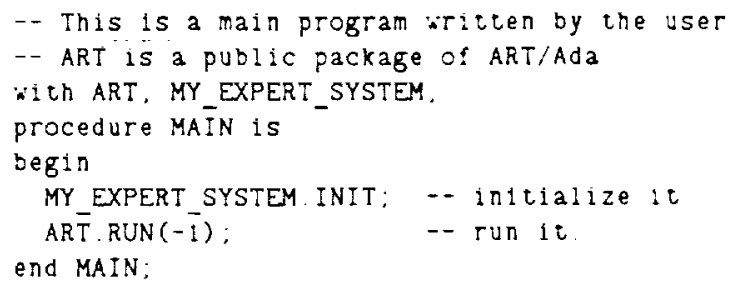

In addition to senerating Ada source code that represents the knowlerlge base, the Ida deployment compiler also generates a callout interlace module that is used to call Ada subprograms from ART Ada ART-MI provides a language to specily the call-out interface for calling Ada subprograms from ART-RI or ART/Ada. The Ada deployment compiler is written in $\mathrm{C}$ and is linked with ART-MI.

\subsection{Ada Call-in and Call-out}

It is common that an expert system application calls out to a procedural language such as $\mathrm{Ada}$ from an expert system shell. Since ART-MI is used to develop an ART/Ada application, it is critical to allow the user to call out to Ada from ART-M.

An expert system application often calls public functions of an expert system tool from a procedural language (e.g. Ada). Since ART-IM is written in C. each public function must be provided with an Ada binding to be called from Ada.

A consistent Ada call-in and call-out interface is provided for both development and deployment environments so that the user-written Ada code runs without modification when it is deployed in Ada after being developed in ART-IM. The ART-IM Ada binding consists of Ada functions that call ART-M's public functions written in C. The specification of public functions in both the ART-IM Ada binding and the ART/Ada runtime kernel is identical

Not all Ada compilers support the feature of calling Ada from C. On VAV/VMS, the DEC Ada compiler can be used because both DEC C and DEC: Ada compilers confirm to the VXIS calling standard. On Unix platforms, the Verdix Ada compiler can be used because it supports this feature well: it is already. being used as an in-house development tool for the ART/Ada project; and it is used by many da programmers on Inix platforms. This restriclion exists only on an ART-IM development plattorm and 
does not prevent the users from porting generaled Na rode and the ART Ada runtime kernel to other Sila compilers and hardware platforms In fact. HeT hala has been already ported to multiple da compiturs including DEC, Nlsys and Verdix and muliple harlware plations such as a VAX/VMS. a iun and an IBN PS:2.

Ala data types supported for the call-in and call-out interfaces are: 3:- bit integer (INTECER_TYPE), 6. bit lloat (FLOAT_TIPE). boolean (BOOLE.NN - TYPE), string (STRING), and an abstract lata type for objects in ART-M (AR'T_OB.JECT). Table 2-1 summarizes the mapping between ART-M, C and Ada data types.

\begin{tabular}{|l|l|l|}
\hline \multicolumn{1}{|c|}{ AR'T-RI } & \multicolumn{1}{c|}{ C } & \multicolumn{1}{|c|}{ Ada } \\
\hline integer & long & INTEGER_TYPE \\
\hline float & double & FLOAT_TIPE \\
\hline boolean & long & BOOLEAN_TIPE \\
\hline string & char $^{*}$ & STRING \\
\hline symbol & char $^{*}$ & STRING \\
\hline art-object & struct $^{*}$ & ART_OBJECT \\
\hline
\end{tabular}

Table 2-1: Data Types for Ada Call-in/Call-out

For example. in order to call out to an Ada function. CALC_AVG, using an ART-IM function, calculate-average. define the following in ART-M":

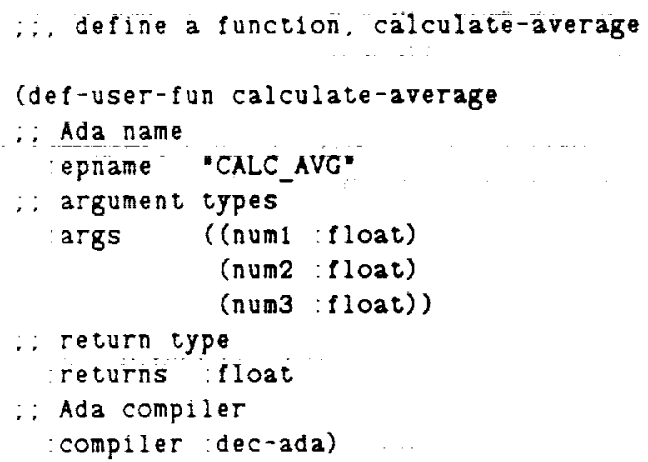

A specification of an Ada package called USER should be also defined as follows:

\footnotetext{
The syotix of ART-IAls provedural language is similar to Common List.
}

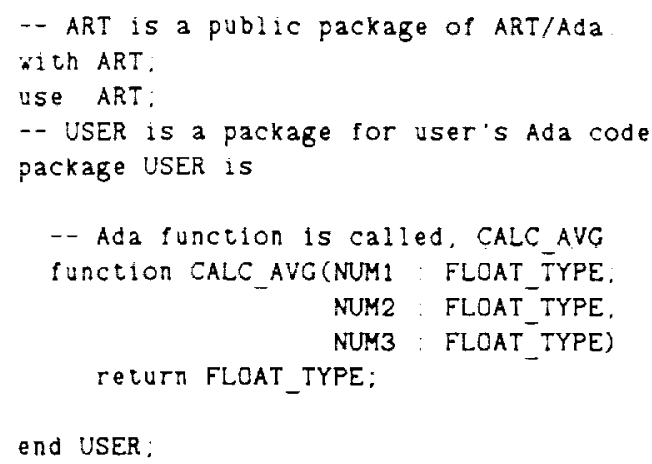

This Ada runction, CALC_AVG, can be called from ART-M as follows:

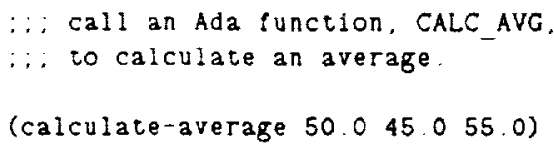

which would return 50.0 .

\subsection{Deployment in Ada}

The methodology for developing an ART,Ada application defines three distinct platforms, some or all of which may be the same:

- an ART-IM development platform with Ada call-in and call-out capability on which an application is actually developed and debugged;

- an Ada compiler platform on which either a self-target compiler or a cross-compiler is used to compile Ada source code; and

- a target platiorm on which an Ada execulable image will be deployed.

The development phase would involve the development of an ART-IM program with Ada code that interfaces with ART-IM through an Ada call-in and call-out interface, which occurs on the ART-RM development phatform. 


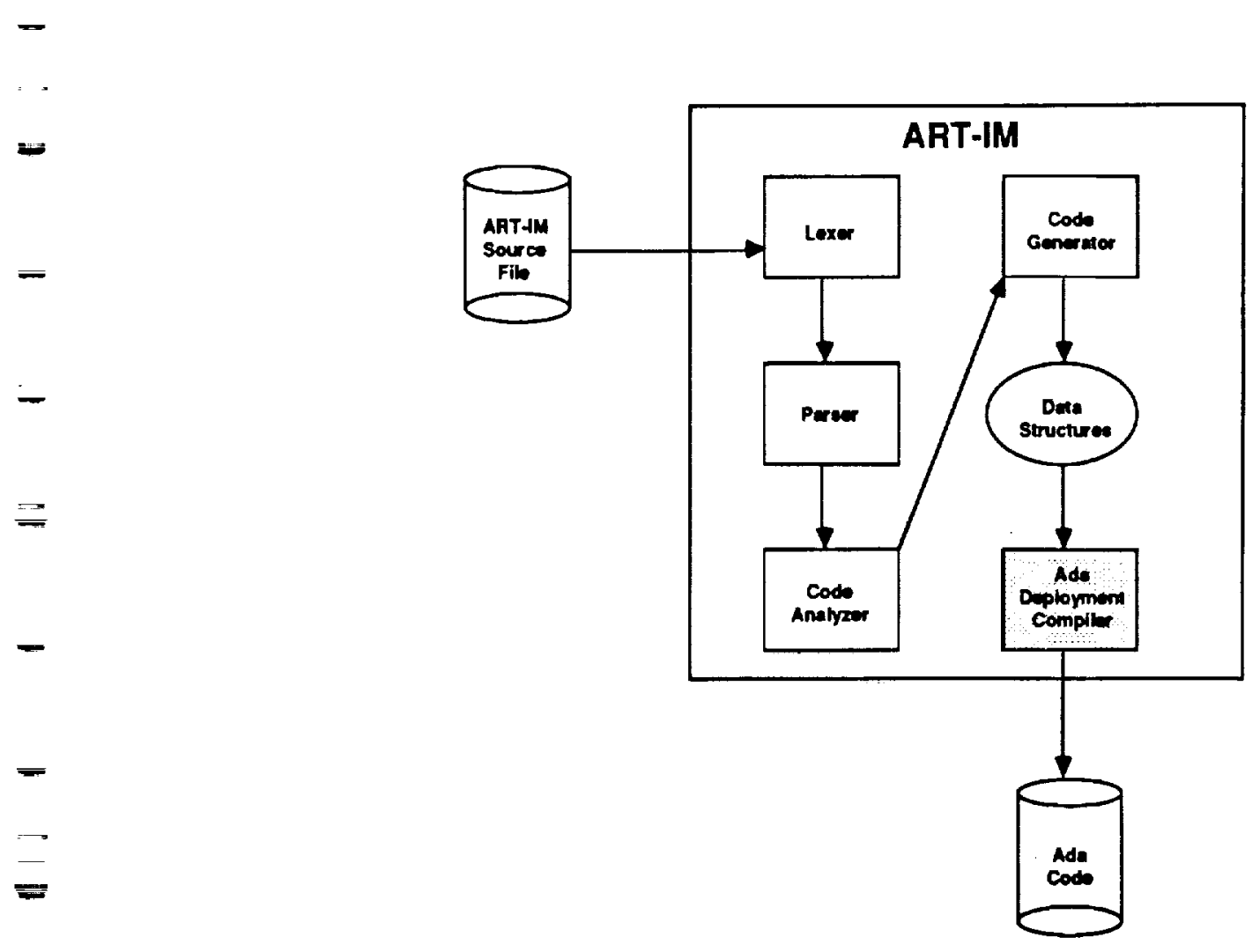

Figure 2-1: Ada Deployment Compiler

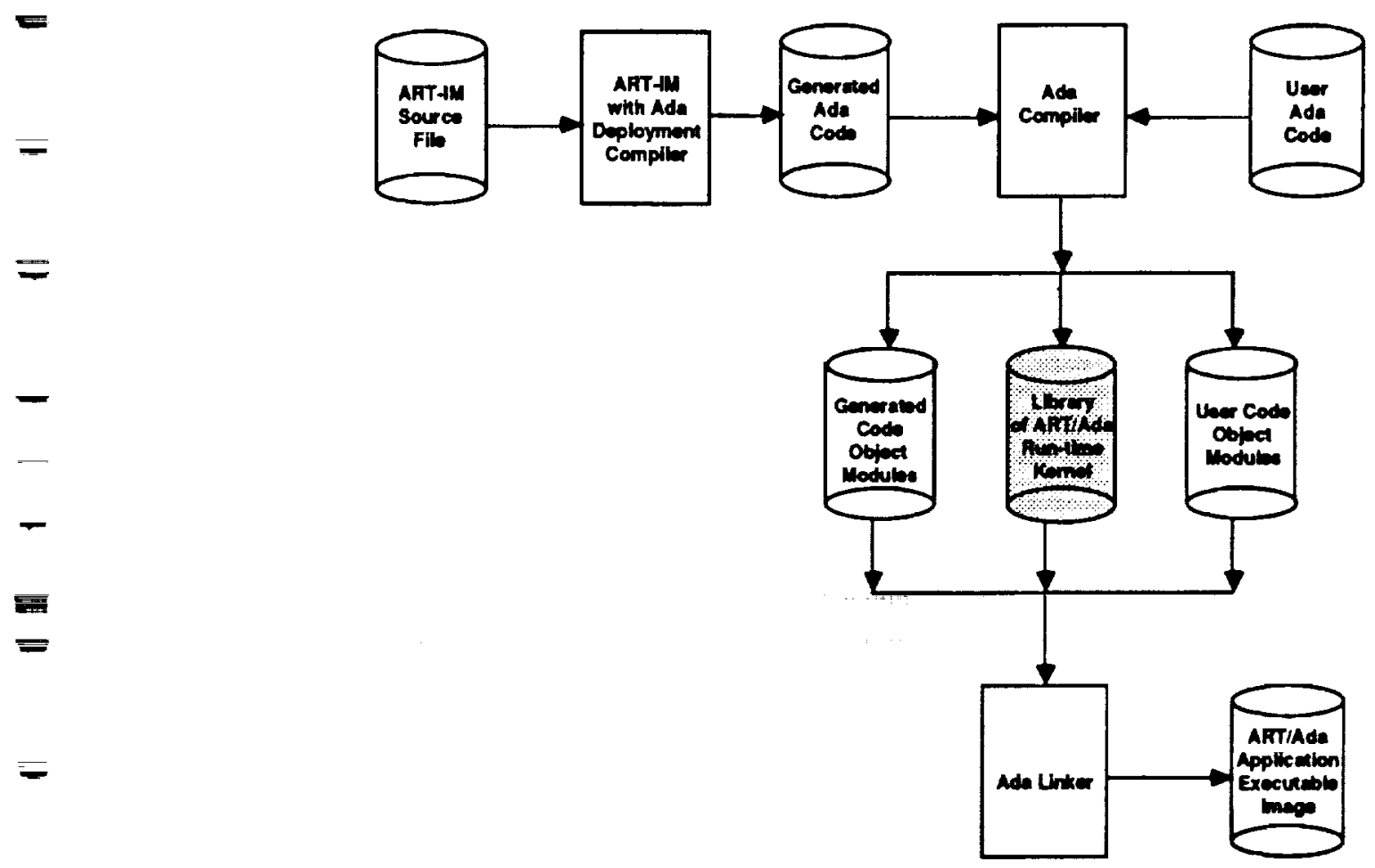

Figure 2-2: Ada Deployment Prosess 
The deployment phase would involve compilation of Ida code generated by the ART-IN Ada deployment compiler and Ada code written by the user, which occurs on the platform where the dia compilet runs. The MPT Ada runtime kernel is provided either in an Alis source colle form or as an dia library that is createrl tasing lhe same da compiler. If the da compiler is a self-target compiler, the Ada execulable image will be deployed on the same platform where the deta compiler runs. If it is a cross-compiler. it will be deployed on the target platform (which may be an on-board computer)

As shown in Figure 2-2, the following steps are needed to deploy an ART-M application in Ada:

1. Develop and debug an application using ART-ML: interactive development environment. If necessary, call out to Ada from $\backslash R T-M I$ using the standard call-out interface, or call into ART-IM from Ada using the Ada binding.

2. Cienerate the Ada code from ART-IM using the Ada deployment compiler. This Ada code is portable to any self-host or cross-compiling Ada compilers. If the Ada compiler platform is different from the ART-IM development platform, the generated Ada code can be moved to the platform on which the Ada compiler runs.

3. Compile the generated Ada code and the uscr-written Ada code using either a selftarget compiler or a cross-compiler into an appropriate Ada library of the ART/Ada runtime kernel.

1. Create an Ada executable image by linking an ida main program.

5. Deploy the Ada executable image on a host compuler or on a target system

\section{Discussion}

\subsection{Performance}

The ART Ada project succeeded in proving that applications of a conventional expert system tool could be deployed in rarious Ada environments whl, afficient ane of lime and space. The preliminary benclumark resilt of the ART Ada protolype shows that the specel and the size of ART Ada protolype is comparable to other tools including C-based tools. alchough it is somewhal slower and larger than ARTMI

The address space limitation of current generation tmbedded processors, such as the MIL-STD-1750A, is I megaword (2 megabytes). within which all soltware systems including the operating system have to run This might be too restrictive for large expert system applications. New generation embedded processors sucl as the 80386 would be more than adequate for expert systems developed using ART/Ada.

While Ada compilers are inproving. they still have not reached the maturity of $\mathrm{C}$ compilers. It has also been observed that both the speed and the size of ART Ada varies up to $30^{\circ}$ depending on which $\mathrm{Ada}$ compiler is used. A recent paper discusses the key technical issues involved in producing high-quality Ada compilers $|\pi|$. As Ada compiler technology advances. ART/Adas performance will improve; we expect to narrow the performance gap between ART$\mathrm{M}$ and ART/Ada.

\subsection{Ada Limitations}

During the reimplementation of the ART-M runtime kernel in Ada, several issues concerning the limitation of Ada language arose.

In order to achieve maximum time and space efriciency. ART-MI has been optimized in ways that are not portable to Ada. For example. the type cast. feature of the $\mathrm{C}$ language has been used both to optimize data structure and to implement an internal memory manager. ART-MI's memory manager maintains its own free list and handles all allocation and deallocation requests from the ART-IM kernel; it allocates large blocks of memory from the system, and then fulfills individual (relatively small) requests for storage from the large blocks. As storage is released. it. is added to an internally maintained free list: the blocks themselves are never released back to the system. There are several advantages 10 this approach: the free space is managed in a common pool by a single facility and is available for allocation 
of arbitrary data types by using the type cast capability in $C$ and $i$ is faster than using syitem routine's for small requests. The stecess of ART-MM's use of type casting relies on other features of the $C^{\prime}$ language definition: there is a direct correspondence betwen addressos and pointer lypes; the mapping between data lypes. insluding structures and arrays, is well defined and strightiforward.

Ada does provide a racility for converting between data types. although this feature has intentionally been made difficult to use. In order to convert from one data lype to another, the generic function unchecked conversion must be instantiated for each conversion required. The existence of a type cast. capability in Ada is insuflicient to implement the ART-IM leatures described above, however. No correspondence is guaranteed between the type SYSTEMIADDRESS and Ada access types. Indeed. on some implementations the underlying representation is different for addresses and access types. The coustraint checking requirements of Ada require that the representation of many objects include descriptor information. The format of these descriptors is not defined by the language. Hence, it is impossible to implement the ART-M style memory manager in tda using unchecked_conversion. Compared to ART-M, this has resulted in some loss of efficiency in ART/Ada that allocates and deallocates memory for each data type directly from or to the Ada runtime system using Ada features, new and unchecked_deallocation.

We also discovered other limitations in Ada that do not exist in $C^{\prime}$ :

- ART-MI has an interpreter (similar to a lisp interpreter) that calls a C function using a $C$ function pointer. To emulate SRT-MI's function call mechanism the Ada deployment compiler automatically generates Ada source code for a procedure called FUNC.ALI, that has a large case statement. This case statement contains all the Ada subprograms that are called rrom an ART Ada application. Each subprogram is assigned with an ID number. To call an Ada subprogram, the procedure FUNCALL is called with a subprogram ID number. While it may callse mainfenance problems. the use of function pointers can provide better performance than lhe ll:se of the tra case statement.

- Bit operations feg bimise exclusire OR, bitwise shift operations, etc.) that may be used to implement efficient hashing algorithms are not provided in dda. They may be implemenled in hal bue only will poor performance

- Because a math library, which is part of the standard C language. is not part of the standard Ada, it is hard to write portable: Ada code that uses math functions.

- Representation specilication is not portable because each ida compiter andior hardware platlorm may use a different memory boundary.

- Variant record is the only Ala data type that can be used to implement C"s union. but it is not as efficient nor liexible.

- Some Ada compilers do not allow calling an Ada program from another language because Ada is a runtime environment as well as a programming language. When it is supported, many restrictions are usually imposed: the main program must be an Ada program, and exceptions and tasking may not be used by the Ada program called from another language.

- In $\mathrm{C}$, conditional compilation facilitated by preprocessor directives (e.g \#define and \#if) allows maintaining a single source file for multiple platforms. In Ada, no such facility exists, and multiple files may have to be maintained for multiple platforns.

- An Ada library system may lead to wasted disk space. For example, an Ada library management system requires duplication of the whole library when the body of a package in the library has dual definitions. In $C$, when functions are defined more than once, they can be simply stored in multiple local libraries while the rest of the program is stored in a main library without duplication. Only one of these multiple local libraries is linked with the 
main library.

- A C-ugle formatting function (e.g. printe. sprinte, elc) is hord, if not impossible, to implement in Ada because the data types of its lunclion arguments are not predetermined.

Various Aa language issues are being studied by several working groups including the Ada Language lsoues llorking Ciroup (ALIVCi) and the Ada Runtime Enviromment Working Group (ARTEW(i). and will be proposed for the Ada $9 \times$ standard $\{1,|2|$. We believe that some of the issues discussed in this paper should also be considered for the Ada $9 \mathrm{X}$.

\subsection{Related Work}

FLAC (Ford Lisp-ida Connection) uses a Lisp environment to develop an expert system application and generates Ada code to be deployed in Ada environments $\{13\}$. Its knowledge base is specified using a graphical representation similar to that of VLSI design (e.g. OR gates and AND gates). FLAC is similar to ART, Ada because its development environment is not implemented in Ada but Ada deployment is supported. The difference is, however, that FLAC's development environment is based on Lisp, while ART/Ada uses that of ART-MM which is written in C. C and Ada development environments coexist on the same hardware platforms more often than Lisp and Ada development environments do. FlAC, lor example, uses a special-purpose Lisp machine for the front-end, and a VAX for the Ada deployment. Both . VRT-MI and ART/Ada can run on the same hardwarc. Another difference is that FL. $\backslash C^{\prime \prime} s$ input is graphics-oriented while ART/Ada is language-oriented. FLAC's knowledge base is precompiled and static. which means that objects may not be added or deleted dynamically at runtime although their values may be changed. This impose major restrictions on the reasoning capability which do not exist in ART-IM and ART, Ada.

CHRONOS is a commercial expert system tool written in Ata that was introduced recently. It is developed and marketed by a French company. Euristic sistems. Is its name implies, it supports temporal reasoning capabilities by time-stamping each fact with tomporal attributes. Currently. little is published about this tool

Another commereial tool is an objectoriencer programming emvimmment alled Classic-ida is, It seems to have its roots in Smallaglk. Flavors and (LOS (Common linp Object Symem). Hhhough Classic-Ada does not -npport rules its object-oriented programming features art similar to APT-IM's object system.

It is reported that several logic-bised took support Prolog in Ada [1], 3, 10]. Nhhough Prolog can be used to implement expert systems. its approach and scope are significantly different lrom expert system tools such as ART-MI. These tools, therefore are not covered in this paper.

\subsection{Future Work}

ARTiAda will be used by several NASA sites to implement prototype expert systems for the Space Station Freedom Program testbeds. This will allow research to understand the potential uses and operational issues of ART Ada.

Our future research effort will be focused on realtime embedded and distributed applications:

- to meet real-time requirements.

- to support distributed environments (e.g parallel processors). and

- to fit into embedded processors.

Real-time requirements are still not very well understood [19]. Siupport lor real-time applications in an expert system tool is usually focused on temporal reasoning capability or on better performance $|4|$. No tool presently available seems to address guaranteed response time. While it is not clear how an expert system tool can satisfy hard realtime requirements by guaranteeing response lime. it: performance could be optimized to satisfy soff realtime requirements $|15|$. Ahlough it is possible to implement temporal ressoning in ART Ada using existing features, it would be straightorward to build

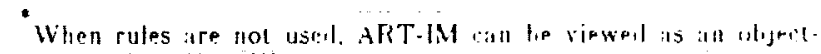
oriented programming environment. 
temporal reasoning capability direculy into ART Ada.

Onc way 10 support paralletism in a Pete-based cxpert sysem lool is to parallelize lhe Pete network ' 8 . This approach may require specialized hardware. Mrother approach is a message-passing architechure that allows mulriple expert systems to communirate asymehromously. This approach can be implemented by developing multiple ART Ada programs and a communications package outside of ART hda leally though, a built-in capability should be provided to support multiple cooperating expert systems that can run as multiple processes on a single processor or as distributed processes on multiple processors. If multiple "knowledge-base packages" are supported in a single program. each package can be deployed as an expert system module that would communicate asynchronously wh other modules through a message passing mechanism that may have to be customized for each software, hardware platiorm. Ada lasking would be ideal for implementing this because it is portable and does not require customization.

Although semiconductor technology is improving very rapidly in the commercial sector, embedded processors are still based on the old technology. Modem operating system features such as virtual memory are not readily available on most on-board computers. The resource requirements on these computers such as processor speed and real memory are quite stringent. It is essential that ART/Ada meet these requirements for the emerging newgeneration embedded processors such as the Intel 80386 . the Intel 80960 , and the MIPS RISC chip.

\section{Acknowledgments}

The authors wish to acknowledge the guidance and support of Chris Culbert. Bob Savely and Bob Brown of NASA Johnson Space Center Mission Planning and Analysis Division, Cireg swietek of NASA Headquarters Space Station Orfice, and Captain Mark Ciersh ol LSAF Technology and Requirements Planning Mark Auburn. Don Pilipovich and Mark Wright of Inference Corpolation contributed to the project.

\section{References}

1. Wa Language Jasues lliorking (iroup. ". Ma Language lswes Working (iroup) (ALIW(i) Mlumes of 17 August 1988". Ida Lellew IS, 1 (Jamuary Februar's 1989)

2. Ida Runtime Environment Horking (iroup.

"Aclivitien of the Ada Rumbene Environment

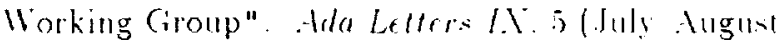
1989).

3. Bobbie, P.O. ADA-PROLOG: An Ada System for Parallel Interpretation of Prolog Programs.

Proceedings of the third Anmual Conference on Artificial Intelligence and Ada. 1987.

4. Burback, R. PROVER: A First-order Logic System in Ada. Proceedings of the third Annual Conference on Artificial Intelligence and Ada. 1987

5. Dzierzanowski, J.M1. et. al. The Authorizer's Assistant: A Knowledge-based Credit Authorization System for American Express. Proceedings of the Conference on Innorative Applications of Artificial Intelligence, AAtI, 1989.

6. Forgy, C.L. "RETE: A Fast Algorithm for the Many Pattern/Many Object Pattern Match Problem". Artificial Intelligence 19 (1982).

7. Ganapathi, M., Mendal, G.O. "Issues in Ada Compiler Technology". Computer 22, ㄴ (February 1989).

8. Gupta. A. Parallelism in Produclion Systems. Pitman Publishing, 1988.

9. Hugh, D.A. "The Future of Flying". M Expert 3, 1 (January 1988).

10. Ice, S., et. al. Raising ALL.IN: Ada Logic-Based Language. Proceedings of the third tunual Conference on Artificial Intelligence and Ada. Ingt

11. Inference Corporation. $A R T$ Iersion 3.2 Reference Manual. Inference Corporation. 1988.

12. Inference Corporation. $-1 K T$-IMI I.5 Referfnce Manual. Inference Corporation. 1989.

13. Jaworski, A., LaVallee, D., Zoch, D. A Lisp-Ada Connection for Expert System Development.

Proceedings of the third Annual Conlerence on Artilicial Intelligence and Ada, 1987. 
14. Lafley T.J. Cox PA. Schmidt JI, Kan.

S.Y. Re;id. I.): "Re:al-Time knowledge-Biand

System". . M Magazinf 9.1 (Spring 1988).

15. Lalley. T, S. Wedzenkamp, Read, J.. Kino. S.

Schunde, I. Intelligent Real-Time Monitoring

Procerdings of lace Viational conference on Artificial

Intelligence. A.A. 1988 .

18. Nakanhima, Y. Baba, T. OHCS. Hydraulic Circuit Design Assistant. Proceedings of the Conference on Innovative Applications of Artificial Intelligence. LLW. 1989.

17. OBrien. J. et. al. The Ford Motor Company Direct Labor Management System. Proceedings of the Conlerence on Innovaltive Applications of Artificial lutelligence. A.M. 198.

18. Software Productivity Solutions. Inc. Classic-Ada Lser Manual. Software Productivity Solutions, Inc, 1988 .

19. Stankovic. J. A. "Misconceptions about RealTime Computing". Computer 21,10 (October 1988). 